\title{
A Novel Cost-Effective Safety Adjunct to Flexible Nasendoscopy During The COVID-19 Pandemic: The Personal Protective Endoscopy Mask (PPEM)
}

\author{
karan jolly ${ }^{1}$, Adnan Darr ${ }^{1}$, Max Osborne $^{1}$, Navdeep Bhamra ${ }^{1}$, and James Barraclough ${ }^{1}$ \\ ${ }^{1}$ Royal Wolverhampton Hospitals NHS Trust
}

May 19, 2020

\section{Introduction:}

In late December 2019, a cluster of pneumonia cases of unknown aetiology were reported in Wuhan, Hubei Province, Central China. The causative agent was subsequently identified as the novel coronavirus (SARSCoV-2, previously known as 2019-nCoV). The coronavirus disease 2019 (COVID-19) was eventually declared as a global pandemic by the World Health Organisation (WHO), having been detected in over 72 countries worldwide, with Europe and the United States now deemed the viral epicentres [1].

Healthcare professionals (HCP) are considered high risk due to a multitude of factors including exposure to higher viral loads [2] and aerosolisation [3,4]. The rising number of deaths amongst healthcare professionals and burden of sick leave secondary to self-isolation, raises significant safety concerns during patient assessment and management. This is particularly of concern when undertaking droplet and aerosol generating procedures (AGP), although the evidence is derived largely from low quality studies [6]. Postulated mechanisms thought to generate aerosol and droplets include laryngeal activity (speech \& coughing), high velocity gas flow, and cyclical opening and closure of distal airway. These have been found to generate particles in a range of sizes, increasing the viral load in confined spaces, and possibly increasing transmission risk.

Recent literature suggests an increased risk posed to otorhinolaryngologists, as diagnostic, interventional and therapeutic procedures involving the upper aerodigestive tract, paranasal sinuses and middle ear exposes HCPs to both direct and indirect transmission of SARS-CoV-2 [7]. At the point of formulating this article, elective clinical workload has been reduced within the United Kingdom. Two-week-wait clinics have continued due to the prevalence and rising incidence of head and neck cancers [8], albeit at a significantly reduced capacity due to lengthened infection control measures.

We describe a novel and cost-effective safety adjunct when undertaking flexible nasendoscopy (FNE) within ward/ outpatient settings.

\section{Technical Description:}

Our technique aims to utilise resources readily available within the healthcare service to construct an easy to use adjunct mask to aid safer FNE. Our device incorporates a standard anaesthetic air cushion face mask, double swivel elbow adapter and a viral filter. In addition, a paediatric mask may be utilised to safely assess for neck stoma patency/ pathology.

Whilst the patient is being examined, the mask is applied and secured with an elasticated band. The soft cushion of the mask conforms to facial contours, creating a comfortable, soft, low-pressure seal around the nose and mouth serving as an additional barrier. The mask comes in a range of appropriate sizes, the transparent structure providing a clear view of the nasal and oral cavities. Masks possess a standard 22F 
port, onto which a double swivel elbow adapter with an in-built $7.6 \mathrm{~mm}$ sealed bronchoscope port (figure 1) is attached. We found the Intersurgical? swivel adaptors best suited for purpose due to their ability to directly attach to the mask, reducing the need for numerous additional components. A viral filter is connected to the second port of the swivel elbow connector (figure 1), allowing respiration, whilst filtering exhaled air to avoid viral transmission. All the 3 components are single-use, and are assembled as a single unit, to form the PPEM (figure 2), which is provided to the patient to don prior to examination (figure 2). Once the patient is comfortable, the sealable bronchoscope port cap is opened, and the FNE is passed through under direct guidance using an appropriate endoscopic camera stack system (figure 3). At this point the mask can be adjusted slightly to allow for appropriate trajectory of the nasoendoscope into the nasal cavity. Any droplets or aerosol generated is contained within the face mask, with the only potential escape points being circumferentially around the FNE entry port site. Having completed the procedure, the nasoendoscope is slowly withdrawn and sent for decontamination, and the bronchoscope port closed. The patient is then advised to retain the mask until having left the room, where it can be disposed of as clinical waste.

In an attempt to estimate aerosol risk reduction using our device, we implemented the use of Betadine solution in a spray applicator (figure 4). The FNE was passed through a tight aperture through a sheet of paper and then passed through the mask as per normal procedure (figure 5). Four sprays were then directed into the mask to simulate aerosol generation, whilst the paper was held an inch away from the bronchoscope port to detect any potential leakage. This process was repeated without the use of a mask, spraying directly onto a sheet of paper from an equal distance to compare the relative reduction of aerosol to the clinician (figure 6). The PPEM demonstrated a significant reduction in potential aerosol exposure to the examiner.

\section{Discussion}

As healthcare devise methods of upscaling service provision back to pre-COVID levels, new methods and techniques are being deployed to maintain productivity and safety of patients and HCPs. Such provisions allow for the anticipated increase in attendances to emergency departments as the public's perceived risk of COVID-19 reduces in the foreseeable future.

Nasendoscopy is a pre-requisite in examining patients in both emergencies and routine outpatient clinic settings and is currently deemed high risk, thus limiting productivity with regards to return to normal activity levels. Our novel approach serves to provide a barrier, which can be utilised in any clinical setting, with readily available components. In our case all components were manufactured by Intersurgical? at a total cost of $£ 1.78$ via NHS supply chain. Additionally, the PPEM provides a superior seal compared to conventional surgical masks, and with certain adaptations, can examine the distal airway through neck stomas.

In addition to serving as a barrier to patients and HCPs, application of the PPEM will also reduce aerosolisation/ droplet exposure within the clinical environment, reducing time for air circulation between patients, thus increasing patient turnover. It has already been approved by our NHS trust for implementation. In addition to the PPEM, we advocate that clinicians should continue to adopt personal protective equipment (PPE) whilst performing the procedure in line with government guidance, particularly whilst more robust and high level evidence is provided.

\section{The PPEM Key points:}

- Air cushion mask combined with swivel elbow connector and viral filter

- Serves as a comfortable additional barrier to droplet/aerosol generation

- Clear view of face mouth and nose throughout entire procedure

- Can be utilised in the assessment of neck stomas

\section{References:}

[1] He F et al. Coronavirus disease 2019: What we know? J Med Virol. 2020 Mar 14. [Epub ahead of print]

[2] Burdorf A et al. The COVID-19 (Coronavirus) pandemic: consequences for occupational health. Scand J 
Work Environ Health. 2020 [Epub ahead of print]

[3] Tran K., Cimon K., Severn M. Aerosol generating procedures and risk of transmission of acute respiratory infections to healthcare workers: a systematic review. PLoS One. 2012;7(4)

10. Boswell C., Longstaff J. Aerosol generating procedures (AGPs) 2019. https://hpspubsrepo.blob.core.windows.net/hps-website/nss/2893/documents/1_tbp-lr-agp-v1.pdf

[4]. O'Neil CA, Li J, Leavey A. Characterization of aerosols generated during patient care activities. Clin Infect Dis. 2017;65(8):1342-1348.

[5] 4. Siddique H., Parveen N., Topping A. Coronavirus deaths of two nurses lead to calls for more protection. The Guardian. 2020 https://www.theguardian.com/society/2020/apr/03/coronavirus-deaths-of-twonurses-lead-to-calls-for-more-protection

[6] Wilson NM et al. Airborne transmission of severe acute respiratory syndrome coronavirus-2 to healthcare workers: a narrative review. Anaesthesia. 2020 Apr 20. [Epub ahead of print]

[7] Couloigner S et al. COVID-19 and ENT Surgery. Eur Ann Otorhinolaryngol Head Neck Dis. 2020 Apr 23. [Epub ahead of print]

[8] Louie KS et al. Trends in head and neck cancers in England from 1995 to 2011 and projections up to 2025. Oral Oncol. 2015 Apr;51(4):341-8. Epub 2015 Jan 22.

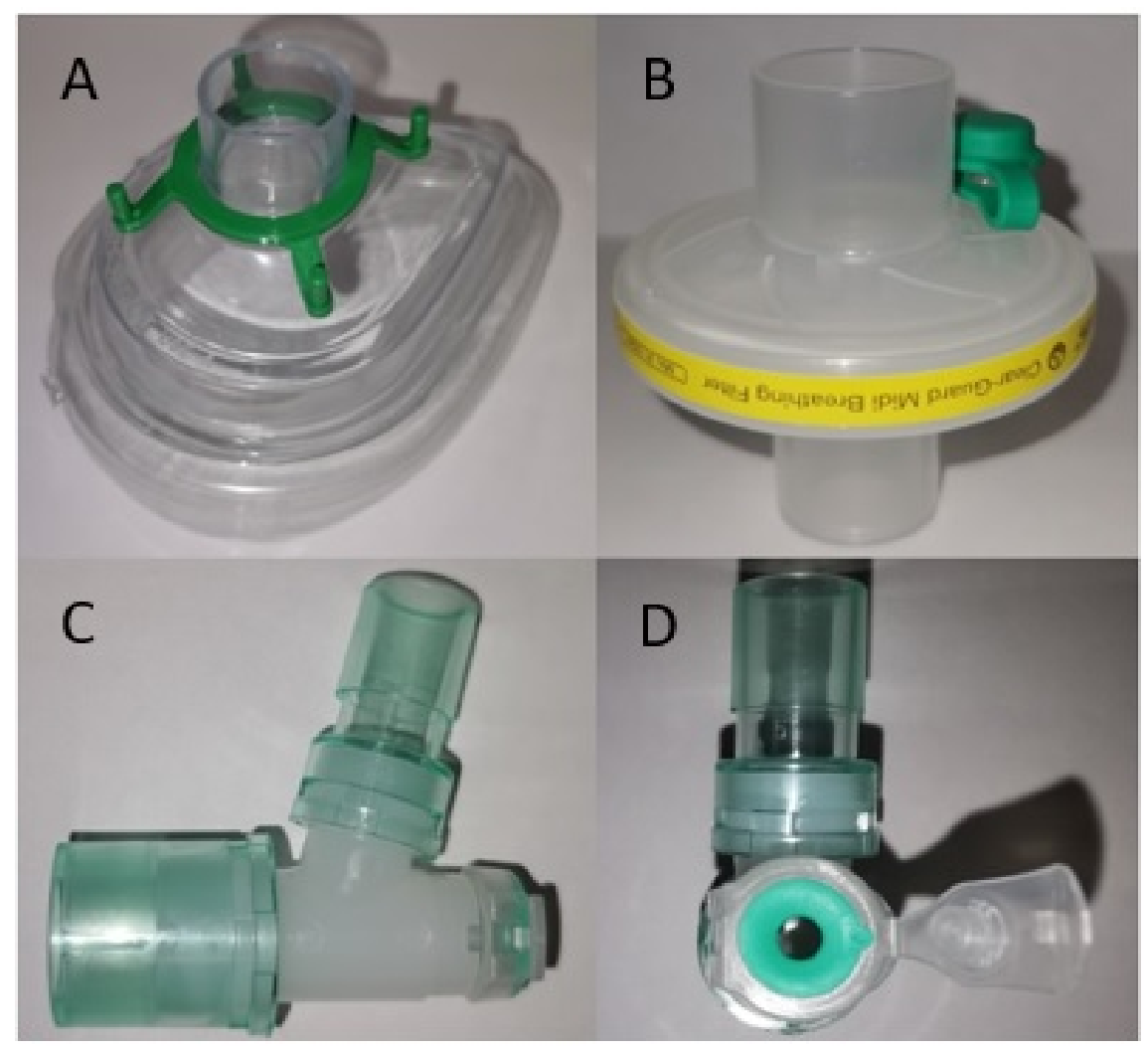



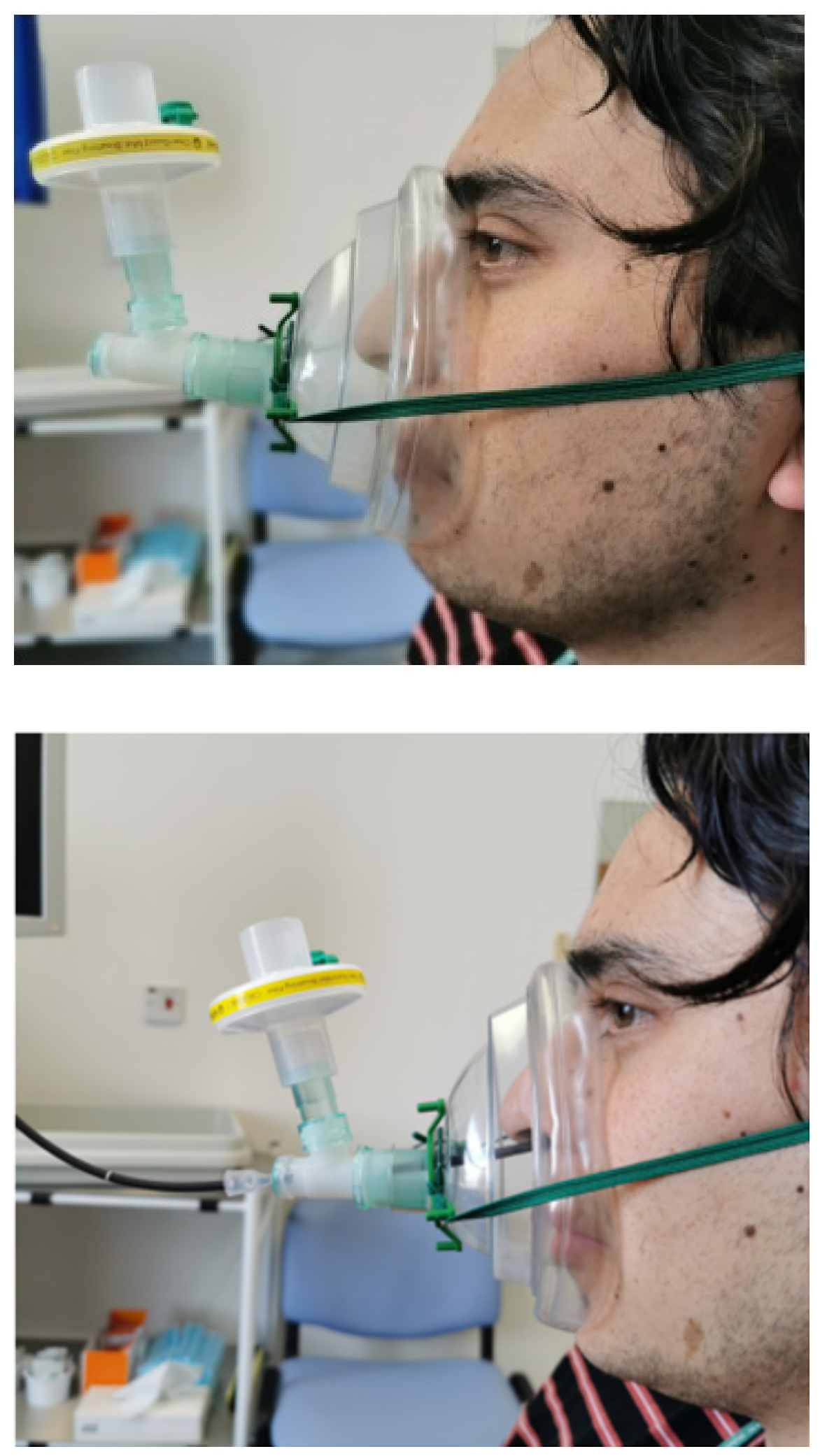

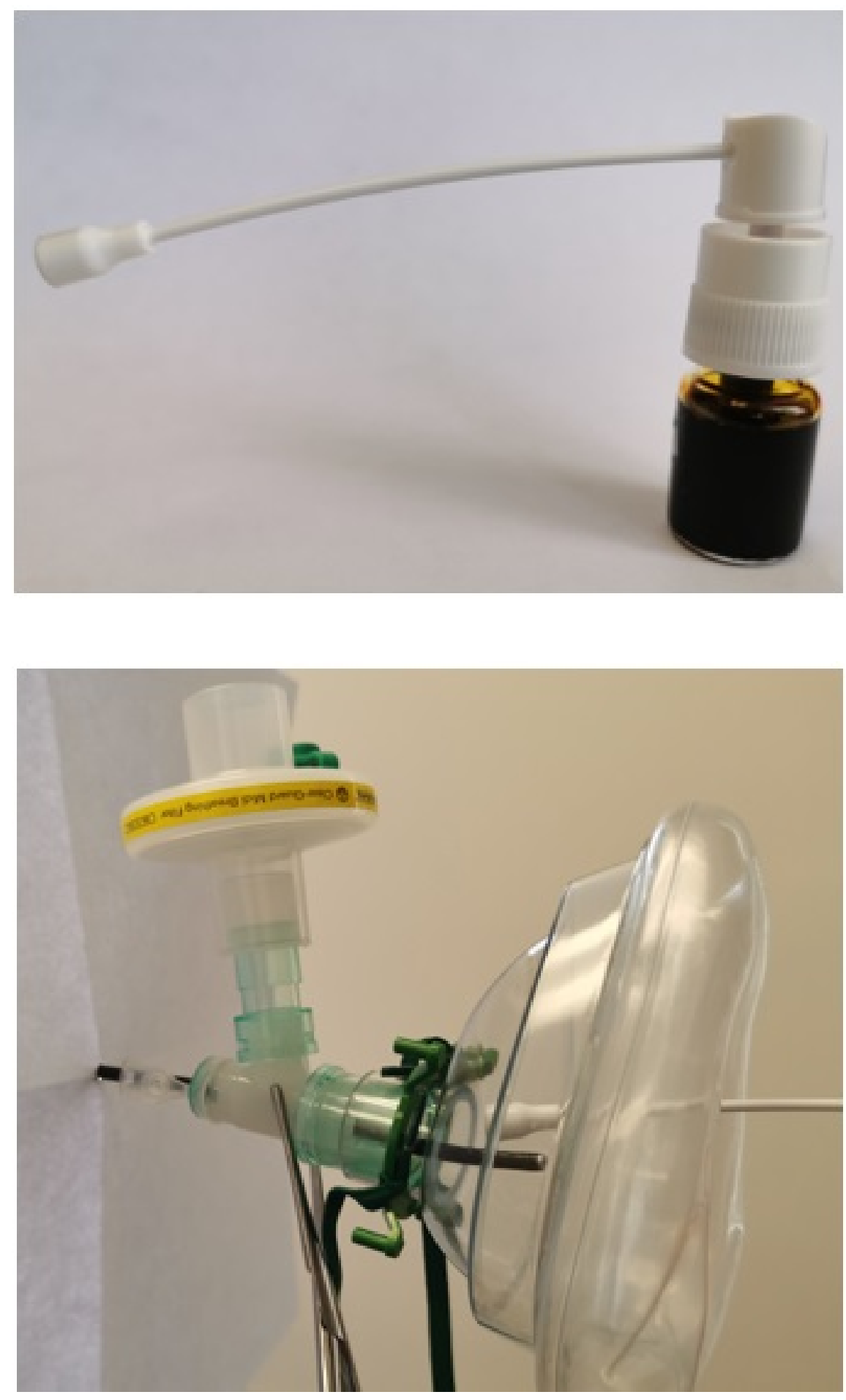


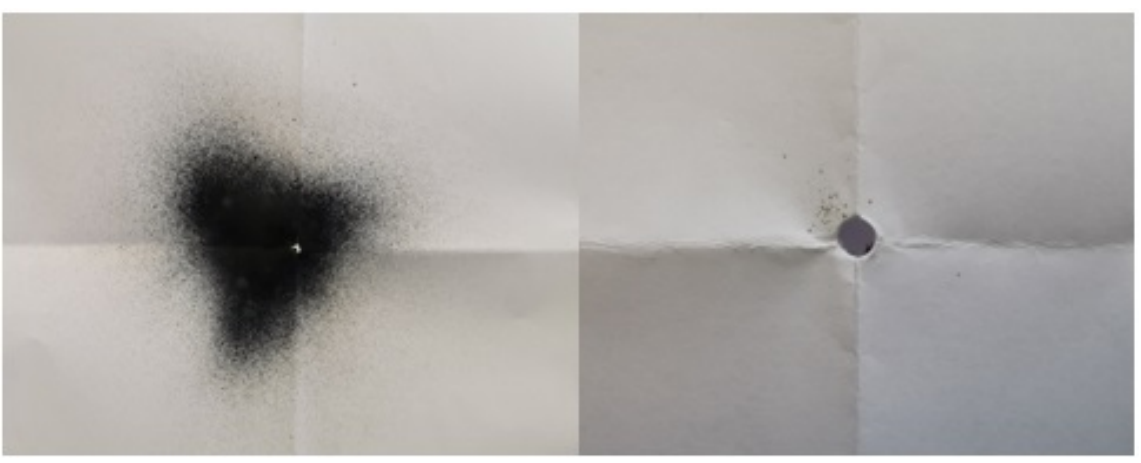

